\title{
Actividad sexual y enfermedad cardiovascular
}

\section{Sexual activity and cardiovascular disease}

\author{
José Manuel Sosa Rosado \\ Cardiólogo Clínica Internacional.
}

\begin{abstract}
Resumen
La prevalencia de las enfermedades crónicas no transmisibles se está incrementando en nuestro medio, ya sea por una mayor esperanza de vida de la población o de adopción de estilos de vida menos saludables, lo que se acompaña de un aumento en la prevalencia de obesidad, hipertensión arterial y diabetes, así como una incidencia mayor de eventos coronarios en pacientes jóvenes. Por lo que, el reinicio de la actividad sexual luego de un evento coronario y el uso de inhibidores de la fosfodiesterasa es un tema que cada vez debemos tratar con más frecuencia con nuestros pacientes y sus cónyuges. Ello tiene un efecto positivo en la salud mental y calidad de vida de nuestros pacientes. En este artículo se plantea las preguntas más frecuentes que debemos resolver los cardiólogos con nuestros pacientes.
\end{abstract}

Palabras clave: Conducta sexual, enfermedad cardiovascular, infarto del miocardio.

\section{Abstract}

The prevalence of non transmissible chronic diseases is increasing among us either because of longer life expectancy of the population or because of the acquisition of unhealthy lifestyles. This goes along with an increase in the prevalence of obesity, arterial hypertension and diabetes, as well as higher incidence in coronary events in young patients. So that restart of sexual activity following a coronary event and use of phosphodiesterase inhibitors are themes that we need to discuss more frequently with our patients and their spouses. This has a positive effect in mental health and quality of life of our patients. In this article the most frequent asked questions that we need to solve as cardiologists with our patients are discussed.

Key words: Sexual behavior, cardiovascular diseases, myocardial infarction.

An Fac med. 2012;73(4):331-4

\section{INTRODUCCIÓN}

En el período 1950-2000, en el Perú el porcentaje de población mayor de 60 años creció de $5,7 \%$ a $7,1 \%$. Este mismo incremento se volverá a repetir en un menor período (2000 al 2015), y se prevé que para el 2050 la proporción de población mayor de 60 años sea de 21,8\% (tres veces lo observado en el año 2000). Los principales problemas que aquejan a estas personas son las enfermedades crónicas degenerativas, donde destacan la hipertensión arterial, diabetes y la cardiopatía isquémica ${ }^{(1)}$. Los recientes avances en la terapia cardiovascular con el advenimiento de la trombólisis y el contar cada vez con más centros donde se puede realizar procedimientos invasivos que permiten la permeabilización de la arteria responsable, ha disminuido la mortalidad y la incapacidad posteriores a eventos coronarios agudos. Ello permite tener pacientes adultos mayores física y sexualmente activos, haciendo imperativo discutir con nuestros pacientes su actividad sexual y sus potenciales riesgos. En las tablas 1 y 2 , se presenta en forma resumida los niveles de evidencia y recomendación para el manejo de estas situaciones, según escalas predefinidas.

El tabú que rodea a la actividad sexual (AS) en general se ve sobredimensionado cuando se involucra la salud, especialmente la del aparato cardiovascular. Los pacientes y sus parejas ven con temor la reanudación de la AS luego de un evento cardiovascular o una cirugía cardiaca. Este desconcierto se exacerba cuando no hay una comunicación fluida entre el médico y su paciente. Uno de los objetivos principales de la rehabilitación cardiovascular es mejorar la calidad de vida de nuestros pacientes y en lo posible su retorno a sus roles sociales previos al evento cardiovascular, tanto dentro de su familia como en la comunidad.

La AS es parte muy importante de la vida de las personas y su continuidad tiene un efecto positivo en la salud mental y calidad de vida. La disminución de la AS es común en los pacientes con enfermedades cardiovasculares y está interrelacionada a la ansiedad y depresión. Por otra parte, la prevalencia cada vez más frecuente de la cardiopatía isquémica en personas de menor edad pone en evidencia la importancia de informar al paciente y su pareja sobre el desempeño sexual tras un evento coronario, informarles sobre los riesgos y resolver todas las preguntas que nos pueden plantear. 
Tabla 1. Grados de recomendación.

\begin{tabular}{ll} 
Grado de recomendación & Definición \\
Clase I & Evidencia y/o acuerdo general de que un determinado procedimiento diagnóstico/tratamiento es beneficioso, útil y efectivo \\
Clase II & Evidencia conflictiva y/o divergencia de opinión acerca de la utilidad/eficacia del tratamiento \\
Clase lla & El peso de la evidencia/opinión está a favor de la utilidad/eficacia \\
Clase Ilb & La utilidad/eficacia está menos establecida por la evidencia/opinión \\
Clase III & Evidencia o acuerdo general de que el tratamiento no es útil/efectivo y en algunos casos puede ser perjudicial \\
\hline
\end{tabular}

Es razonable que los pacientes con enfermedad cardiovascular (ECV) con esperanzas de reiniciar su AS deben ser primero evaluados cuidadosamente para determinar su riesgo de complicaciones cardiovasculares. Los pacientes con ECV inestable descompensados, y/o ECV severa o sintomática, deben diferir la AS hasta que su condición sea estabilizada (clase III; nivel de evidencia C) ${ }^{(2)}$.

En pacientes cuya capacidad de ejercicio o riesgo cardiovascular es desconocido, la prueba de esfuerzo (PE) puede ser útil para evaluar su capacidad de ejercicio y desarrollo de síntomas, signos de isquemia, cianosis, hipotensión o arritmias.

Las preguntas más frecuentes de nuestros pacientes se refieren a cuándo reiniciar la AS, relaciones extraconyugales, disfunción eréctil (DE) y el uso de inhibidores de la 5 fosfodiesterasa (5PDE).

\section{¿CUÁNDO REINICIAR LAS ACTIVIDADES SEXUALES?}

\section{Post síndrome coronario agudo}

Los estudios realizados en hombres jóvenes heterosexuales han demostrado que la actividad sexual con una pareja habitual es comparable a una actividad física leve a moderada, donde la frecuencia cardíaca raramente excede los 130 latidos por minuto y la presión arterial raramente excede los 170 $\mathrm{mmHg}$. Equivale a un gasto energético entre 3 a 4 equivalentes metabólicos (METS) (2-4) (un MET es la cantidad de oxígeno consumida por el organismo en reposo y equivale a $3,5 \mathrm{~mL} \mathrm{O} / \mathrm{kg} /$ $\mathrm{min})$. Las relaciones sexuales pueden iniciarse transcurridos entre una a dos semanas posteriores al alta hospitalaria. Todo dependerá de la capacidad funcional del paciente en la prueba de esfuerzo, y si es o no negativa a isquemia. Los pacientes que pueden superar los 5 METS en una PE sin presentar angina o equivalentes anginosos, alteraciones electrocardiográficas, arritmias, pueden reiniciar sus actividades sexuales con su pareja estable $^{(2,5,6)}$ (clase IIa, nivel de evidencia C) ${ }^{(2)}$.

Las ventajas de realizar una PE con personal capacitado están en relación a su larga historia de uso y validación de sus resultados, y su costo bajo; fácilmente accesibles y reproducibles en la evaluación de nuestros pacientes, nos permiten valorar su capacidad funcional y tolerancia al ejercicio, lo cual podemos extrapolar a su capacidad de mantener AS.
Los pacientes que han tenido una angioplastia exitosa por vía radial están hábiles para reanudar su AS en pocos días posteriores al procedimiento ${ }^{(2,4)}$, si no hay complicaciones en el sitio de acceso vascular (clase IIa, nivel de evidencia C) (2). Los pacientes con isquemia residual deben ser aconsejados a reiniciar su AS según los resultados de su PE (clase IIb, nivel de evidencia C) ${ }^{(2)}$. En los pacientes que han sido sometidos a cirugía de revascularización coronaria, se aconseja el reinicio de la AS entre las seis a ocho semanas posteriores a la cirugía ${ }^{(2,4)}$ (clase IIa, nivel de evidencia B) ${ }^{(2)}$.

Hay que evitar posiciones poco habituales, en la pareja que requiera una gran demanda de esfuerzo físico para mantener la postura. Es recomendable aconsejar la utilización del posicionamiento habitual en la pareja, o que la pareja tome la posición superior y el comando de la actividad en las primeras ocasiones, lo cual hace ganar confianza al paciente. Según un estudio de Bohlen ${ }^{(3)}$, los valores alcanzados durante la actividad sexual con el hombre en la posición superior fueron equivalentes a 3,3 METS, y de 2,5 METS cuando la mujer ocupó la posición superior, respectivamente. La auto estimulación alcanza un valor de 1,8 METS y la estimulación por la pareja 1,7 METS ${ }^{(2,7)}$.

Tabla 2. Niveles de evidencia.

\section{Nivel de evidencia Definición}

A Datos procedentes de múltiples ensayos clínicos con distribución aleatoria o metaanálisis
B Datos procedentes de un único ensayo clínico con distribución aleatoria o de grandes estudios sin distribución aleatoria
C


Así mismo, debe evitarse tener relaciones sexuales tras la ingesta moderada de alcohol o comidas copiosas.

\section{Insuficiencia cardíaca}

Para muchos pacientes con insuficiencia cardíaca (IC), es más importante mejorar su calidad de vida (incluyendo la AS) que la mejoría de su supervivencia. La optimización del tratamiento médico y la rehabilitación cardiovascular y el ejercicio regular incrementan la posibilidad de una AS segura y satisfactoria en pacientes con enfermedad cardiovascular (clase IIa, nivel de evidencia B).

Recomendaciones: Tomar en cuenta la clasificación de la Asociación del Corazón, de Nueva York ${ }^{(8)}$, que determina cuatro clases de IC sobre la base de la valoración subjetiva que hace el médico durante el interrogatorio clínico, y fundamentado sobre la presencia y severidad de la disnea y/o angina.

- Clase I: no se experimenta limitación física al movimiento, no aparecen síntomas con la actividad física rutinaria, a pesar de haber disfunción ventricular (confirmada, por ejemplo, por ecocardiografía);

- Clase II: ligera limitación al ejercicio; aparecen los síntomas con la actividad física diaria ordinaria - por ejemplo subir escaleras-, resultando en fatiga, disnea, palpitaciones y angina, entre otras. Desaparecen con el reposo o la actividad física mínima.

- Clase III: marcada limitación al ejercicio. Aparecen los síntomas con las actividades físicas menores, como el caminar, y desaparecen con el reposo.

- Clase IV: limitación muy severa, incapacidad para realizar cualquier actividad física. Aparecen los síntomas aún en reposo.

1. Se señala que la AS es razonable para pacientes con IC clase I-II (clase IIa; nivel de evidencia B) ${ }^{(2)}$.
2. La AS no es aconsejada a pacientes con IC descompensada o clases IIIIV, hasta que su condición sea estabilizada y óptimamente manejada (clase III; nivel de evidencia C) ${ }^{(2,4)}$.

Los programas de rehabilitación cardíaca mejoran la calidad de vida del paciente con IC y pueden mejorar la tolerancia al ejercicio, favoreciendo su AS.

\section{Arritmias}

La muerte durante el coito es extremadamente rara en la población general. Hay pocos estudios sobre la ocurrencia de arritmias durante la AS en pacientes con historia conocida de arritmias. El riesgo de arritmias ventriculares durante la AS no parece ser mayor que durante el ejercicio físico en una $\mathrm{PE}{ }^{(4)}$.

\section{Relaciones extraconyugales}

No es raro que tengamos que tratar este tema con nuestros pacientes. Generalmente, se presenta cuando ya ha pasado cierto tiempo del accidente coronario o en la primera ocasión en que el paciente acude a consulta sin la compañía de la pareja habitual. Este tipo de relaciones generalmente ocasiona un mayor gasto energético.

En estas relaciones sexuales influyen otros factores, como la tensión que se genera al practicar sexo en relaciones extramaritales o el caso de hombres de edad avanzada que tienen relaciones extraconyugales con parejas más jóvenes, y generalmente se acompañan de comidas copiosas, ingesta de licor e inhibidores de la 5PDE.

El riesgo de infarto de miocardio asociado a actividad sexual es bajo, pero mayor en las relaciones extramaritales. En el estudio de Ueno ${ }^{(9)}$, en 5559 pacientes con muerte súbita, se determinó que solo 0,6\% (34 personas) de ellas fue durante la actividad sexual. La proporción de muertes en el contexto de relaciones extramaritales fue de $70 \%$ (24 personas); solo dos fueron mujeres. Parzeller ${ }^{(10)}$ encontró en un estudio retrospectivo médico legal que la incidencia de muerte cardiovascular fue en 48 casos $(0,16 \%)$ de 26901 autopsias; de estas, 25 fueron por infarto de miocardio, 12 en un primer infarto y 13 en un segundo infarto; 36 (75\%) de las muertes fueron en relaciones extramaritales, y solo 3 de las 48 fueron mujeres.

Es importante establecer que el grado de excitación también tiene rol importante. Cantwell ${ }^{(11)}$, en un estudio con monitoreo electrocardiográfico de 24 horas, encontró que, con la pareja usual, la FC subía de $72 / \mathrm{min}$ a $92 / \mathrm{min}$, mientras que con una pareja eventual ascendía de 96/min a 150/min. El incremento de la ectopia ventricular y otras arritmias puede deberse a un mayor estimulación simpática, similar a la acontecida durante situaciones emocionales intensas. El consenso de la American Heart Association ${ }^{(4)}$ refiere que hay información mínima sobre la AS en pacientes en riesgo de arritmias ventriculares, no observándose un incremento importante en la actividad ectópica

Por otra parte, diversos autores asocian un mayor esfuerzo cardiaco con relaciones extramaritales, ya que de una demanda corporal total de oxígeno durante la AS marital, entre 3 a 4 METS, se puede pasar en la AS extramarital o una AS vigorosa a un aumento del gasto de energía entre 5 a 6 METS. En la práctica clínica, los pacientes capaces de alcanzar 6 METS en la PE, sin síntomas cardiovasculares, pueden generalmente, pero no siempre, realizar actividades sexuales sin experimentar síntomas cardiovasculares ${ }^{(12,13)}$.

\section{Disfunción eréctil (DE) y enfermedad cardiovascular}

Recientemente, los cardiólogos somos consultados con mayor frecuencia por esta patología, bastante frecuente en los pacientes coronarios. Ello se debe a una fisiopatología común. Este grupo demográfico de pacientes con DE se superpone considerablemente al de los pacientes con cardiopatías, ya que los factores de riesgo para la DE son los mismos que para la cardiopatía is- 
quémica. Estos incluyen hipertensión arterial, diabetes, tabaquismo, anormalidades lipídicas (bajos niveles de HDL colesterol e hipercolesterolemia), obesidad, sedentarismo ${ }^{(14,15)}$. Dado el menor diámetro de las arterias peneanas, es comprensible que puedan verse afectadas antes que las arterias coronarias, ya sea por la disfunción endotelial, por la alteración de la vasodilatación o por un proceso obstructivo arteriosclerótico. Por lo que se considera a la DE como un síntoma centinela de la enfermedad coronaria ${ }^{(16,17)}$.

Por otro lado, también debemos considerar los efectos colaterales de algunos medicamentos cardiológicos, como los betabloqueadores, diuréticos y los cuadros de depresión.

Así mismo, debido al auge y popularidad del uso de los bloqueadores de la 5 PDE en el tratamiento de la DE, es imperioso evaluar y aconsejar a nuestros pacientes sobre la posibilidad o no de utilizar dichos fármacos.

Los 5 PDE5 inhibidores son generalmente seguros y efectivos para el tratamiento de la DE en pacientes hipertensos, enfermedad coronaria estable (clase I; nivel de evidencia A) (2). Recientes estudios, en los que los inhibidores de 5 PDE fueron administrados a pacientes coronarios conocidos, realizando pruebas de esfuerzo no se observó un incremento del umbral de angina o isquemia asociada al ejercicio ${ }^{(18-21)}$. También, cuando se administró sildenafil a pacientes coronarios durante cateterismos cardiacos, no hubo evidencia de que se precipitara isquemia (22). El sildenafil causa una reducción de la presión arterial sistólica $(8$ a $10 \mathrm{mmHg}$ ) y diastólica $(5-6 \mathrm{~mm} \mathrm{Hg})$ transitoria y modesta; existe un efecto pico de la droga en una hora después de ingerida, con efectos vasodilatadores periféricos. La interacción medicamentosa más seria es la de los inhibidores de la $5 \mathrm{PDE}$ con los nitratos (dinitrato de isosorbida, mononitrato de isosorbida, entre otros), medicación usada muy frecuentemente por los pacientes cardíacos. Su interacción produce una vasodilatación severa que lleva hipotensión marcada y a veces fatal ${ }^{(23-26)}$, por lo cual no se recomienda la administración de los bloqueadores de la 5-PDE en pacientes que reciben nitratos (clase III; nivel de evidencia B) (2).

\section{CONCLUSIONES}

A todos los pacientes (y sus cónyuges) que presentan enfermedades cardiovasculares, debe informárseles en forma individualizada sobre el reinicio de su vida sexual, tal como les informamos sobre su vuelta a su centro de labores o participación en programas de ejercicios. Se recomienda a los pacientes con una PE mayor a 5 METS y sin síntomas, que pueden reiniciar su AS siempre con la iniciativa y mayor esfuerzo por parte de su cónyuge. No se recomienda relaciones extraconyugales por las razones expuestas.

En cuanto a quienes presenten DE, informarles sobre la contraindicación absoluta del uso de nitratos conjuntamente con inhibidores de la 5PDE.

\section{REFERENCIAS BIBLIOGRÁFICAS}

1. Análisis de la situación de salud en el Perú. Ministerio de Salud, DGE. Agosto 2010. Capítulo 1:9-22.

2. Levine GN, Steinke EE, Bakaeen FG, Bozkurt B, Cheitlin M, et al. Sexual activity and cardiovascular disease: a scientific statement from the American Heart Association. Circulation. 2012;125(8):105872.

3. Bohlen JG, Held JP, Sanderson O, Patterson PR. Heart rate, rate pressure product, and oxygen uptake during four sexual activities. Arch Intern Med. 1984;144:1745-8.

4. Taylor HA. Sexual activity and the cardiovascular patient: guidelines. Am J Cardiol. 1999;84:6N-10N.

5. DeBusk RF. Evaluating the cardiovascular tolerance for sex. Am J Cardiol. 2000;86(2A):51F-56F.

6. Drory Y. Sexual activity and cardiovascular risk. Eur Heart J Suppl. 2002;4 (suppl H):H13-H18.

7. Thorson Al. Sexual activity and the cardiac patient. Medscape Cardiology. 2003 Le Jacq Communications, Inc.

8. Criteria Committee, New York Heart Association. Diseases of the heart and blood vessels. Nomenclature and criteria for diagnosis, 6th ed. Boston: Little, Brown and Co, 1964:114.

9. Ueno M. The so-called coition death. Jpn J Leg Med. 1963;17:330-40.

10. Parzeller M, Raschka C, Bratzke H. Sudden cardiovascular death during sexual intercourse: results of a legal medicine post morten study from 1972-1998. Letters to the editor. European Heart J. 2001;22:610-6
11. Cantwell, JD. Sex and the heart. Med Aspects Human Sexuality. 1981;15:14-23.

12. Stein R, Barlem C. Sexual activity and heart. Arquivos Brasileiros de Cardiologia. 2006;86(1):

13. Jackson G. Sexual intercourse and stable angina pectoris. Am J Cardiol. 2000;86:35F-37F.

14. Virag R, Bouilly P, Frydman D. Is impotence an arterial disorder? A study of arterial risk factors in 440 impotent men. Lancet. 1985;8422:181-4.

15. Feldman HA, Johannes CB, Derby CA, Mohr $B A$, Araujo $A B$, et al. Erectile dysfunction and coronary risk factors: prospective results from the Massachusetts Male Aging Study. Prev Med. 2000;30:328-38.

16. Vannappagari S. Should erectile dysfunction be considered as a marker for acute myocardial infarction? Int J Impot Res. 2004;16:350-3.

17. Ponholzer A, Temml C, Obermayr R, Wehrberger C, Madersbacher $\mathrm{S}$.Is erectile dysfunction an indicator for increased risk of coronary heart disease and stroke? Eur Urol. 2005;48:512-8.

18. Arruda-Olson AM, Mahoney DW, Nehra A, Leckel M, Pellikka PA, et al. Cardiovascular effects of sildenafil during exercise in men with known or probable coronary artery disease. A randomized crossover trial. JAMA. 2002;287:719-25.

19. Fox KM, Thadani U, Ma PT, Nash SD, Keating Z, et al. Time to onset of limiting angina during treadmill exercise in men with erectile dysfunction and stable chronic angina: effect of sildenafil citrate. Circulation. 2001;107:II-601.

20. Thadani U, Smith W, Nash S, Bittar N, Glasser S, et al. The effect of vardenafil, a potent and highly selective phosphodiesterase- 5 inhibitor for the treatment of erectile dysfunction, on the cardiovascular response to exercise in patients with coronary artery disease. J Am Coll Cardiol. 2002;40:2006-12.

21. Patterson D, MacDonald TM, Effron MB, Emmick JT, Mitchell M, et al. Tadalafil does not affect time to ischemia during exercise stress testing in patients with coronary artery disease. Circulation. 2002;106(Suppl II):II-330.

22. Hermann HC, Chang G, Klugherz BD, Mahoney PDI. Hemodynamic effects of sildenafil in men with severe coronary artery disease. N Engl J Med. 2000;342:1662-6.

23. Webb DJ, Freestone S, Allen MJ, Muirhead GJ. Sildenafil citrate and blood-pressure lowering drugs: results of drug interaction studies with an organic nitrate and a calcium antagonist. Am J Cardiol. 1999;83(Suppl 5A):21C-28C

24. DeBusk R, Drory Y, Goldstein J,Jackson G.Kaul S, et al. Management of sexual dysfunction in patients with cardiovascular disease: recommendations of the Princeton Consensus Panel. Am J Cardiol. 2000;86:175-81.

25. Cheitlin MD, Hutter AM, Brindis RG, Ganz P, Kaul S, et al. ACC/AHA Expert Consensus Document. Use of sildenafil (Viagra) in patients with cardiovascular disease. J Am Coll Cardiol. 1999;33:273-82.

26. Schwartz.B, Levine L, Comstock G, Stecher VJ, Kloner RA. Cardiac uses of phosphodiesterase-5 inhibitors. J Am Coll Cardiol. 2012;59(1):9-15.

Artículo recibido el 23 de junio de 2012 y aceptado para publicación el 26 de agosto de 2012.

Correspondencia:

Dr. José Manuel Sosa Rosado

Correo electrónico: sosa_josemanuel@yahoo.com 